Revue des patrimoines

\title{
La culture matérielle comme support de la mémoire historique : l'exemple des naufragés de Tromelin
}

Thomas Romon et Max Guerout

\section{OpenEdition}

Édition électronique

URL : http://journals.openedition.org/insitu/10182

DOI : 10.4000/insitu. 10182

ISSN : 1630-7305

Éditeur

Ministère de la culture

Référence électronique

Thomas Romon et Max Guerout, «La culture matérielle comme support de la mémoire historique : l'exemple des naufragés de Tromelin », In Situ [En ligne], 20 | 2013, mis en ligne le 15 février 2013, consulté le 19 avril 2019. URL : http://journals.openedition.org/insitu/10182 ; DOI : 10.4000/ insitu.10182

Ce document a été généré automatiquement le 19 avril 2019.

\section{(c) (1) $(9)$}

In Situ Revues des patrimoines est mis à disposition selon les termes de la licence Creative Commons Attribution - Pas d'Utilisation Commerciale - Pas de Modification 4.0 International. 


\title{
La culture matérielle comme support de la mémoire historique : l'exemple des naufragés de Tromelin
}

\author{
Thomas Romon et Max Guerout
}

1 Avec beaucoup de cynisme, cette histoire pourrait se résumer au fait que pour sauver leur vie, des Français, très chrétiens, ont été contraints d'abandonner leur cargaison. Ils n'ont pu embarquer que le strict nécessaire sur l'embarcation de fortune qui leur permettra de quitter l'île sur laquelle ils ont fait naufrage : des vivres et leur chapelle portative. Cette île s'appelait encore l'île des sables. C'était le 27 septembre 1761. Et leur cargaison était constituée par des esclaves, 160, achetés en fraude à Madagascar trois mois plus tôt. Pour gagner l'île de France (aujourd'hui Maurice), ils avaient choisi une route inhabituelle, les faisant passer par le nord. Dans la nuit du 31 juillet, leur bâtiment, l'Utile, se fracassa contre les récifs qui ceinturent l'île de Tromelin. Au matin, 123 membres de l'équipage sont sains et saufs, mais prisonniers de ce minuscule morceau de terre perdu en mer, mesurant moins d'un kilomètre carré. Ils n'ont ni eau, ni vivres, ni abri et aucun moyen de quitter l'île. Concernant les esclaves, un petit peu moins d'une centaine a survécu au naufrage. On s'organise, on récupère sur l'épave : des vivres, barils d'eau, de vin, d'eaude-vie, de farine, de lard et de bœuf, ainsi que des outils, du bois, des voiles... Tout ce que l'on peut! Et si on rationne l'équipage, rien n'est donné aux esclaves. On creuse un premier puits, sans succès, puis un deuxième et on trouve enfin de l'eau. On peut alors songer à en donner aux esclaves. Entre temps, une trentaine d'entre eux est décédée. Avec des mâts et des voiles, on construit deux campements, l'un pour les noirs, l'autre pour les blancs. Puis on construit une embarcation, baptisée la Providence, pour rejoindre Madagascar ; un four pour cuire les biscuits du retour...

Les 123 membres de l'équipage repartiront abandonnant les esclaves, car il n'y a pas assez de place pour tout le monde.

3 Tout ceci est fort bien documenté. Par le journal personnel de Keraudic, l'écrivain du bord, un document manuscrit conservé au service historique de la Défense à Lorient ${ }^{1}$. Par 
des imprimés de colportage, dont un fut imprimé à Bordeaux ${ }^{2}$. Et par plusieurs cartes manuscrites, précisant la position de l'épave, des camps, des puits, du four, de la forge et du chantier de construction de la Providence. Autant dire que cette histoire était connue : celle du naufrage et du salut de l'équipage, mais aussi celle de l'abandon sur l'île d'un grand nombre de noirs javas ${ }^{3}$.

Cependant, quinze années s'écoulèrent avant le sauvetage de ces derniers. Il y a à cela plusieurs raisons : la difficulté de trouver l'île et d'y aborder, mais surtout la volonté de l'administration et particulièrement du gouverneur de l'île de France, d'étouffer cette affaire. Ce n'est qu'à partir de 1775 , les mœurs ayant évolué, qu'on enverra des navires tenter le sauvetage. Et c'est le 29 novembre 1776 que la Dauphine, commandée par l'enseigne de vaisseau de Tromelin, recueille à son bord les survivants : sept femmes et un enfant de huit mois (fig. $\left.\mathbf{n}^{\circ} \mathbf{1}\right)$.

Figure 1

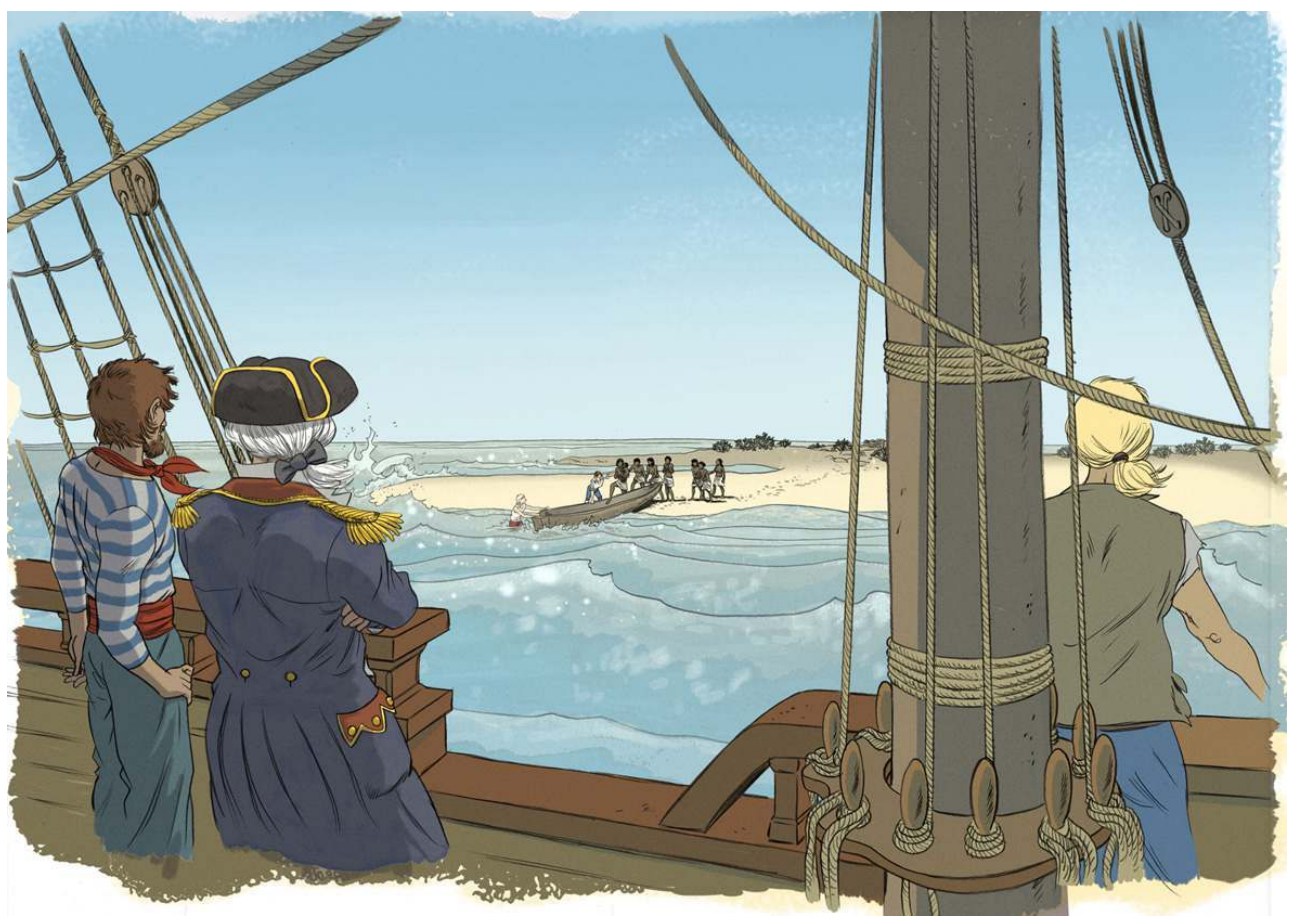

Le Sauvetage des derniers survivants, illustration de Sylvain Savoia. Le 29 novembre 1776, l'enseigne de vaisseau de Tromelin, à bord de la Dauphine, supervise l'embarquement des sept rescapées et du bébé.

(c) S. Savoia-Dupuis-matin\&soir film.

5 Ici encore, cette histoire est bien documentée par les rapports de l'enseigne de vaisseau de Tromelin et de l'intendant Maillard. Mais pas de trace du récit des rescapés. Seul a été retrouvé le compte rendu qu'en donne le Journal historique et politique des principaux événements des différentes Cours de l'Europe de 1777. Finalement, c'est ce séjour qui est le moins bien renseigné. Comment vécurent-ils pendant leurs quinze années d'isolement?

6 La mémoire de cet événement s'est dissipée, lentement, avec le temps, ressurgissant de temps en temps, au gré de la visite de quelques privilégiés sur l'île de Tromelin. Tous, à la vue des vestiges encore présents, parlent des naufragés, mais avec de moins en moins de détails à mesure que ces derniers disparaissent. Les choses changent en 1953 lorsque s'implante sur l'île une station météorologique. Les météorologues qui s'installent au 
milieu des ruines de l'habitat des esclaves en connaissent l'histoire. Ils font un relevé succinct des vestiges encore en élévation, les photographient et effectuent ce que l'on pourrait qualifier de première fouille archéologique. La description qui en est faite, incluse dans le rapport de la mission de reconnaissance, est des plus sommaires. En outre, l'installation de la station sur le lieu même de l'habitat des naufragés lui est fatale. En réutilisant les matériaux encore visibles pour leurs propres constructions, ils en gomment les dernières traces.

7 Il est intéressant de noter combien la mémoire d'un événement dépend de son support matériel. Et combien la perte de ce support est responsable d'une véritable amnésie historique, qui compte tenu de notre penchant naturel à l'oubli, nous touche trop souvent ; les événements qui se sont déroulés à Tromelin en sont l'illustration. En 2006, les derniers témoins matériels de cette histoire sont les canons et l'ancre de l'Utile qui sont visibles depuis le rivage (fig. $\mathbf{n}^{\circ} \mathbf{2}$ ). Il faut y joindre un canon qui trône devant la station météo. Il fut récupéré par les Français en 1761, pour donner l'alerte au cas où un navire viendrait à croiser au large de l'île. Ces objets focalisent l'attention sur le naufrage, qui grâce aux documents laissés par l'équipage rescapé, est aussi fort bien documenté. Par contre, du séjour des Malgaches après le départ des Français, on ne sait plus grand-chose si ce n'est qu'on en sauva huit après quinze ans d'oubli.

Figure 2

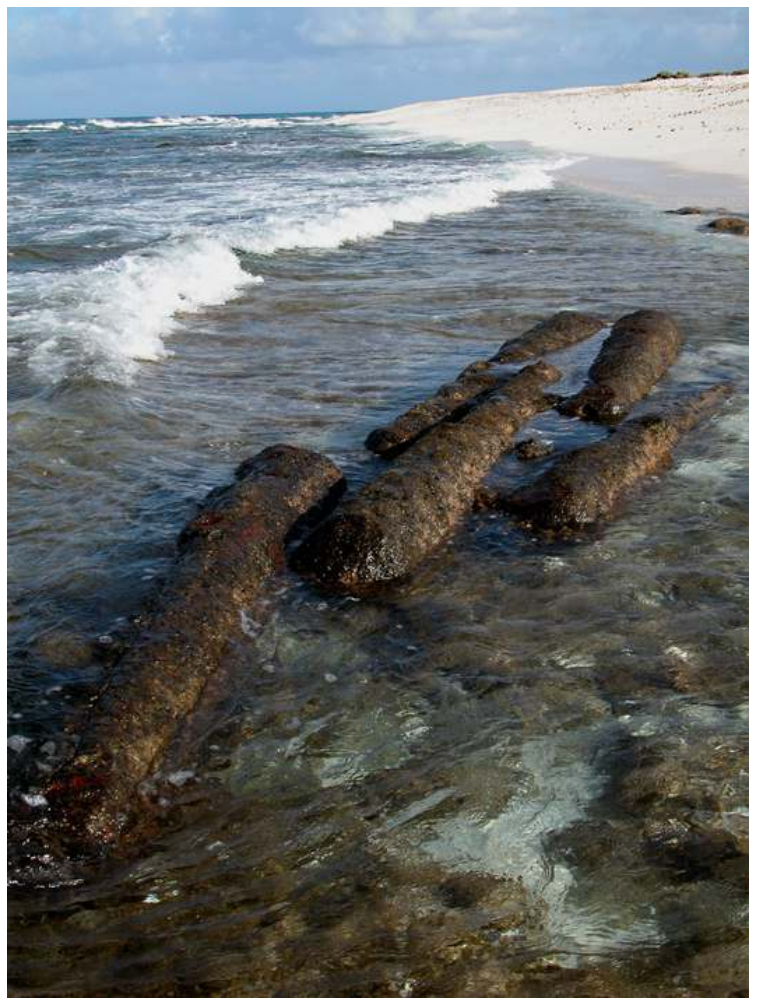

Les vestiges de L'Utile. Cinq canons jetés sur le rivage.

(c) J.-F. Rebeyrotte-Gran.

8 Contre vents et marées et après avoir longuement exploré les sources historiques concernant ce naufrage, Max Guérout, responsable des missions archéologiques menées à Tromelin ces six dernières années, tient à la bipolarité des recherches sur le terrain : d'un côté la mission sous-marine, de l'autre la mission terrestre. L'intérêt de cette démarche 
n'est absolument pas partagé. Il ne doit plus rien rester, lui dit-on, sur cette île balayée par les cyclones. Les résultats montreront le contraire ${ }^{4}$. Du navire, il ne reste que les éléments les plus lourds, les canons (fig. $\mathbf{n}^{\circ} \mathbf{3}$ ), les ancres, les boulets, le lest. Les investigations sous-marines démontrent que l'épave échouée sur la côte occidentale de Tromelin est bien celle de L'Utile. Le mobilier et la dynamique du naufrage correspondent point pour point aux données historiques.

Figure 3

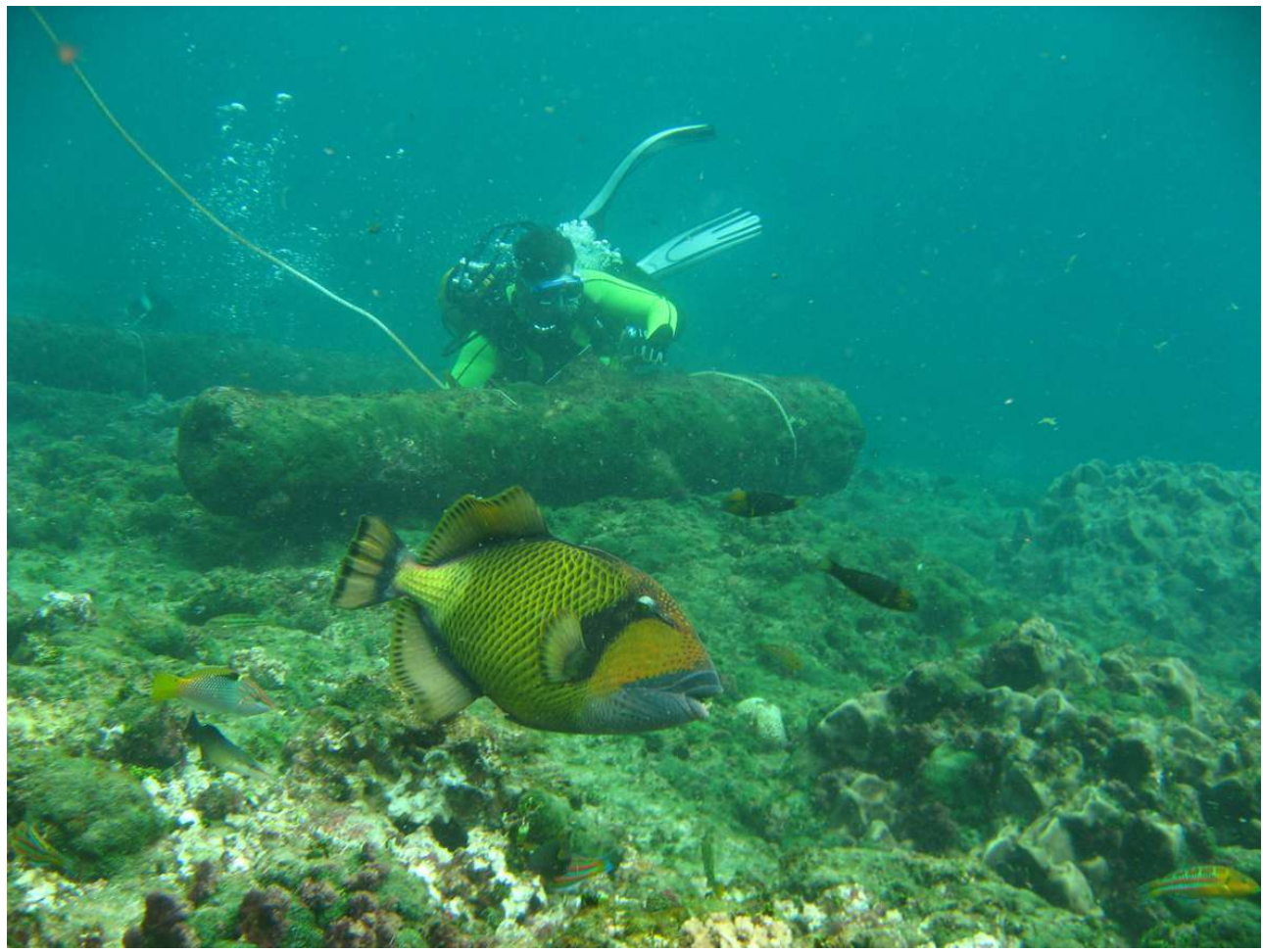

Les investigations sous-marines. Mesure des canons.

(C) J.-F. Rebeyrotte-Gran.

Et sur terre?

Les vestiges de l'occupation durant la présence des Français sont fugaces. Celle-ci fut très courte: à peine deux mois et les campements furent installés dans une zone où la sédimentation est faible. Les puits sont très probablement sous la piste d'aviation et la forge sous les bâtiments de la station météorologique. Par contre, l'emplacement du four a été très vite localisé. Grâce à la carte de 1761, et grâce au travail des tortues, qui depuis deux-cent-cinquante ans creusent leurs nids et remontent les éléments enfouis à la surface. Jamais ces indices n'avaient été interprétés à leur juste signification. Des sondages menés à cet endroit ont permis de retrouver la localisation précise du four. Ils ont aussi permis de fouiller et d'échantillonner les niveaux d'occupation de 1761 contemporains du séjour des Français. Ils sont situés à $60 \mathrm{~cm}$ sous le sol actuel et ont principalement livré des restes de consommation (de la faune aviaire) mêlés à des rejets de vidange de foyer. Par ailleurs, la prospection systématique de l'arrière plage entre l'épave et le four, au moyen de sondages de $1 \mathrm{~m}^{2}$, a permis de mettre en évidence une zone interprétée comme une aire de stockage et de débitage des débris de l'épave avant leur acheminement vers le four, la forge ou le chantier naval. 
11 Les vestiges de l'occupation après le départ des Français sont quant à eux enfouis sous $1 \mathrm{~m}$ de déblais accumulés depuis les années 1950 (fig. $\mathbf{n}^{\circ}$ 4). Il faut creuser la terre, comme on se creuse la mémoire. Dépasser les souvenirs les plus récents, ceux d'un temps, béni pour les archéologues du futur, où la gestion des déchets n'était pas raisonnée. Pour arriver sur une couche de sable de plage d'un blanc immaculé, mesurant de 20 à $50 \mathrm{~cm}$ d'épaisseur, accumulé durant les cent-soixante-quinze ans où l'homme fut le plus souvent absent de l'île. Cette couche est très importante, car elle scelle les niveaux archéologiques. Nous sommes en quelque sorte en présence d'une «petite Pompéi de l'Océan Indien ", à la seule différence qu'ici, la catastrophe fut antérieure au dépôt sédimentaire. À la base de cette couche se trouve la photographie du 29 novembre 1776, jour du sauvetage des sept survivantes et de leur enfant. Certes tout n'y est pas révélé. Mais pour qui sait les déchiffrer, ces vestiges sont la clef permettant d'accéder au quotidien des naufragés. Ce qu'ils ont mangé, comment ils l'ont cuisiné, comment ils se sont protégés des intempéries, comment ils se sont organisés et comment ils ont reconstruit une société. Car tout indique qu'ils n'ont pas fait que subir les éléments, ils les ont combattus et ont laissé leur empreinte sur l'île.

Figure 4

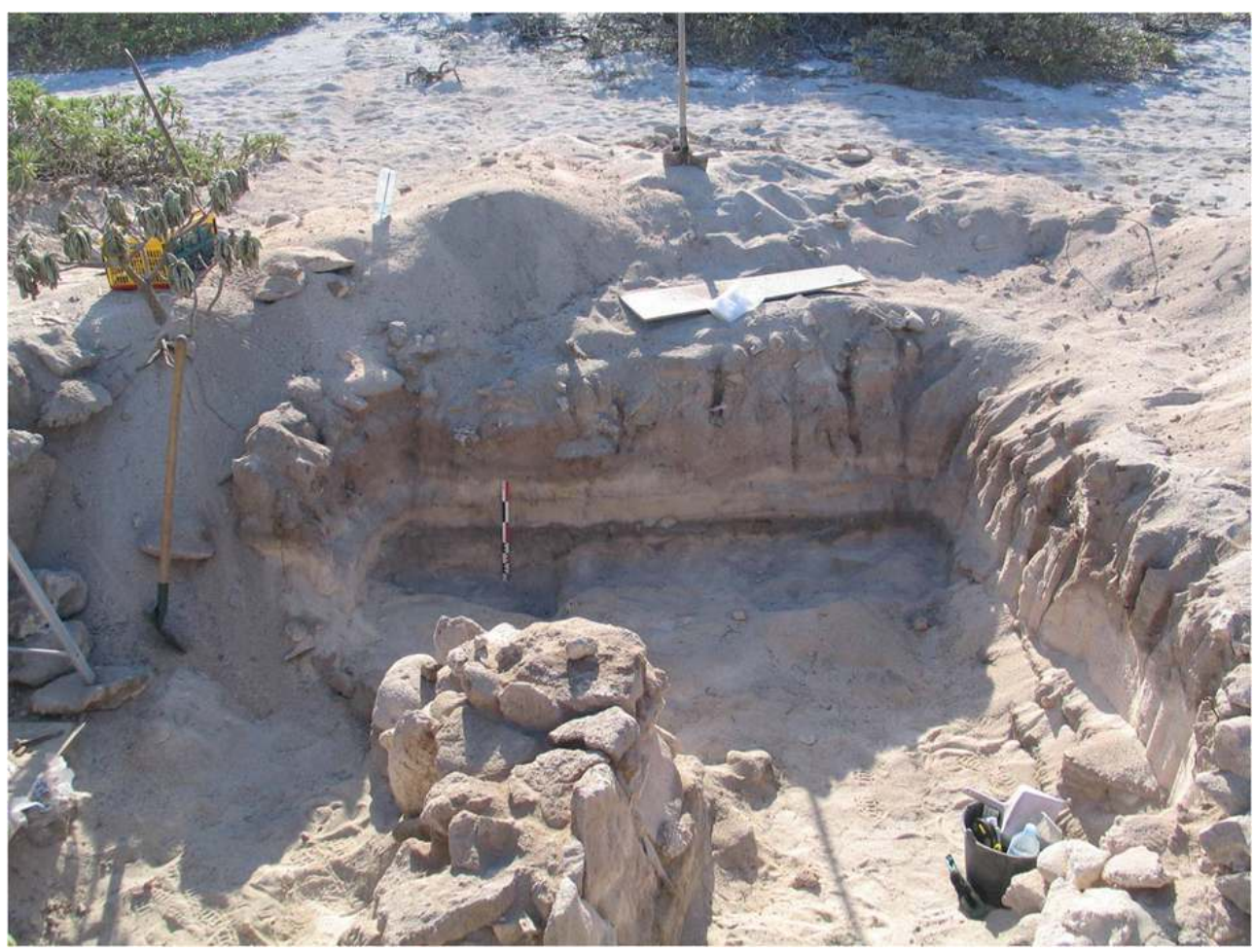

La stratigraphie du site : 1) l'implantation de la station météorologique ; 2) couche de sable de plage ; 3) l'occupation des naufragés entre 1761 et 1776 ; 4) sable de plage.

(c) T. Romon-Gran.

12 Tout d'abord en construisant des abris permettant de protéger des éléments naturels hommes et biens (fig. $\mathbf{n}^{\circ}$ ) ). Ce sont de petites cellules de 2,3 à 2,7 $\mathrm{m}$ de longueur par 1,3 à 2,4 $\mathrm{m}$ de largeur, imbriquées les unes contre les autres. Les murs sont construits en pierres sèches, à l'aide de blocs de corail et de plaques de grès de sable. Ces matériaux sont présents à la surface de l'île, mais préférentiellement dans sa partie sud, alors que l'habitat est au nord. Un parement régulier délimite l'intérieur de l'unique pièce de 
chaque bâtiment. Si une certaine homogénéité dans la forme et les grandes lignes de construction peut être mise en évidence, dans le détail, chacune est unique et semble indiquer soit une évolution dans le temps, soit plus probablement une édification où chaque groupe apporte sa touche. Il y a donc un aspect à la fois collectif et individuel, peut-être le reflet de ce groupe hétérogène constitué d'esclaves prélevés en divers endroits de Madagascar et peut-être même d'Afrique. Mais le groupe est uni dans une même lutte pour sa survie.

Figure 5

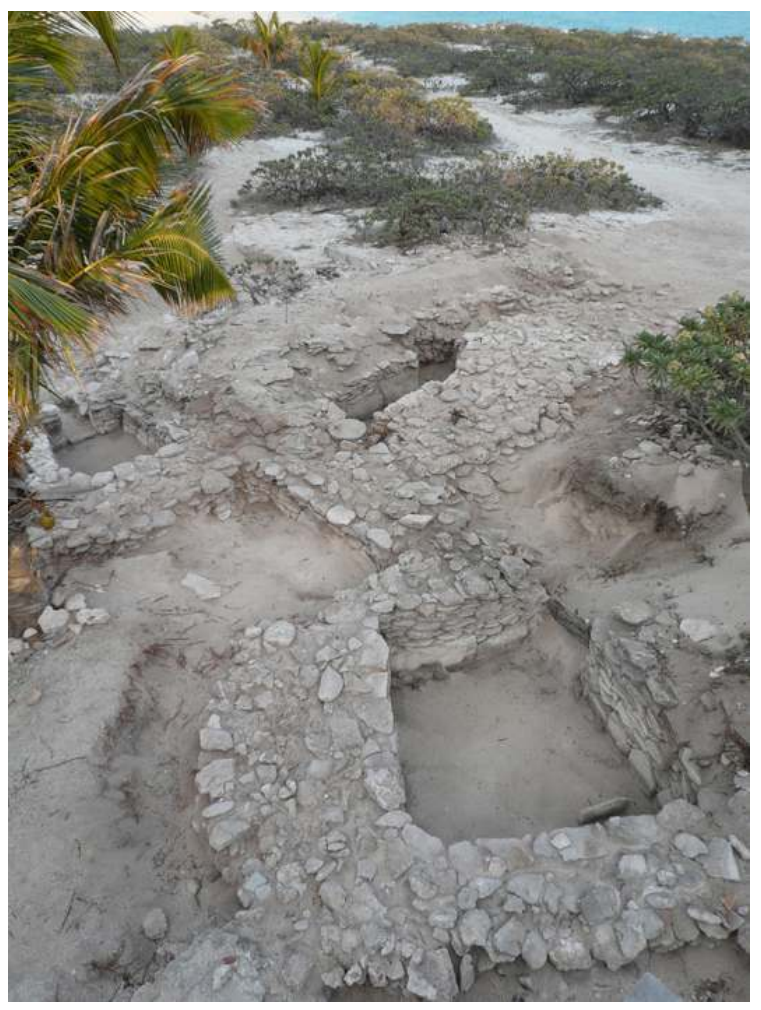

Les bâtiments mis au jour en 2008. Les murs, dont la partie supérieure est arasée, sont très épais et délimitent une pièce unique de très petite dimension.

(c) J.-F. Rebeyrotte-Gran.

13 Par ailleurs, l'habitat malgache répond à des impératifs religieux, en particulier liés à l'orientation, qui font de l'abri domestique un microcosme mettant en relation l'ordre de l'univers et celui de la sociétés. Ces impératifs ne sont pas respectés ici. Les constructions sont orientées dos aux vents dominants et l'espace de vie extérieur est sous le vent des bâtiments. À ceci s'ajoute l'utilisation de la pierre. À Madagascar, elle est traditionnellement réservée aux tombeaux et aux morts. L'habitat des vivants est construit en matière périssable, végétaux et terre, toutefois absents de l'île.

Ces survivants ont aussi laissé leur empreinte dans le mobilier qu'ils ont abandonné. La plupart de ces objets sont utilitaires et proviennent de l'Utile. Soit qu'ils aient été utilisés tels quels, soit qu'ils aient été transformés par les naufragés. Il s'agit surtout d'ustensiles culinaires (fig. $\mathbf{n}^{\circ} \mathbf{6}$ ) et d'outils. Ceux qui ont été récupérés sur l'épave sont des trépieds, des contenants en cuivre (casseroles et gamelles), des crochets, des hameçons, des haches, des marteaux et des briquets et leurs pierres à feu. Ceux qui ont été fabriqués à partir d'éléments provenant de l'épave sont des récipients en cuivre et en plomb, des 
cuillères en cuivre, des outils (clous affutés, penture transformée en hache, cadène en marteau et cheville à grosse tête en enclume) et une pierre de lest réutilisée en affutoir. Ceux qui ont été transformés à partir d'éléments glanés sur l'île sont des coquillages aménagés, une cuillère taillée dans une porcelaine, et une louche dans une conque de triton. Les objets non utilitaires sont deux bracelets en cuivre, cependant trouvés dans les déblais de construction de la station météorologique, mais dont la provenance est incontestablement des niveaux XVIII ${ }^{e}$ siècle, et trois aiguilles en cuivre aux manches gravés et qui sont interprétées comme des pointes démêloirs.

Figure 6

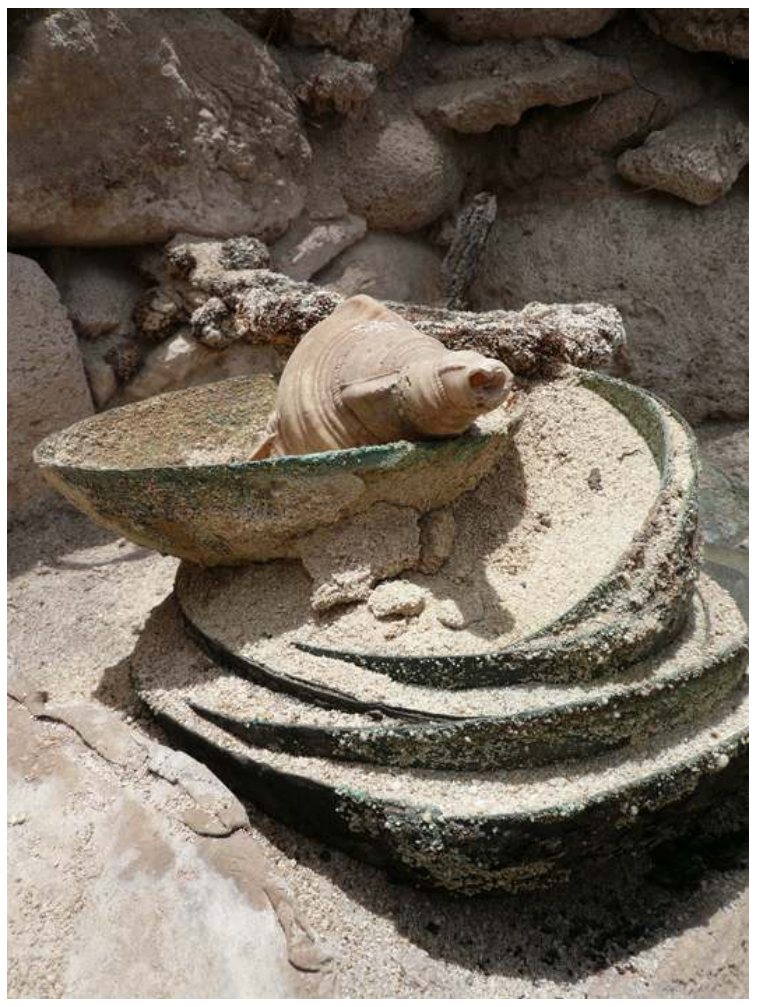

Le mobilier des naufragés. Ici des ustensiles de cuisine (récipients en cuivre) surmontés d'un coquillage évidé. Ils étaient rangés à l'intérieur d'un des bâtiments construits par les naufragés après le départ des Français.

(c) J.-F. Rebeyrotte-Gran.

Enfin, les naufragés ont laissé leur empreinte au travers de leurs rejets, principalement des restes consommés. La couche d'occupation archéologique est très riche en os fragmentés. Il s'agit principalement d'oiseaux et de tortues, les restes de poissons étant très rares et la consommation de coquillages non avérée. L'animal le plus consommé est la sterne fuligineuse. Elle nichait sur l'île au moment du naufrage, comme l'attestent à la fois les récits des Français et les restes d'immatures et de femelles en période de ponte retrouvés dans les niveaux XVIII ${ }^{\mathrm{e}}$ siècle. Les sternes étaient consommées grillées et découpées à l'aide d'outils tranchants (fig. $\mathbf{n}^{\circ} 7$ ). Les extrémités distales des ailes sont absentes. Elles ont été brisées avant cuisson. Elles ont été prélevées peut-être pour utiliser les plumes pour un usage artisanal, comme le tissage. 


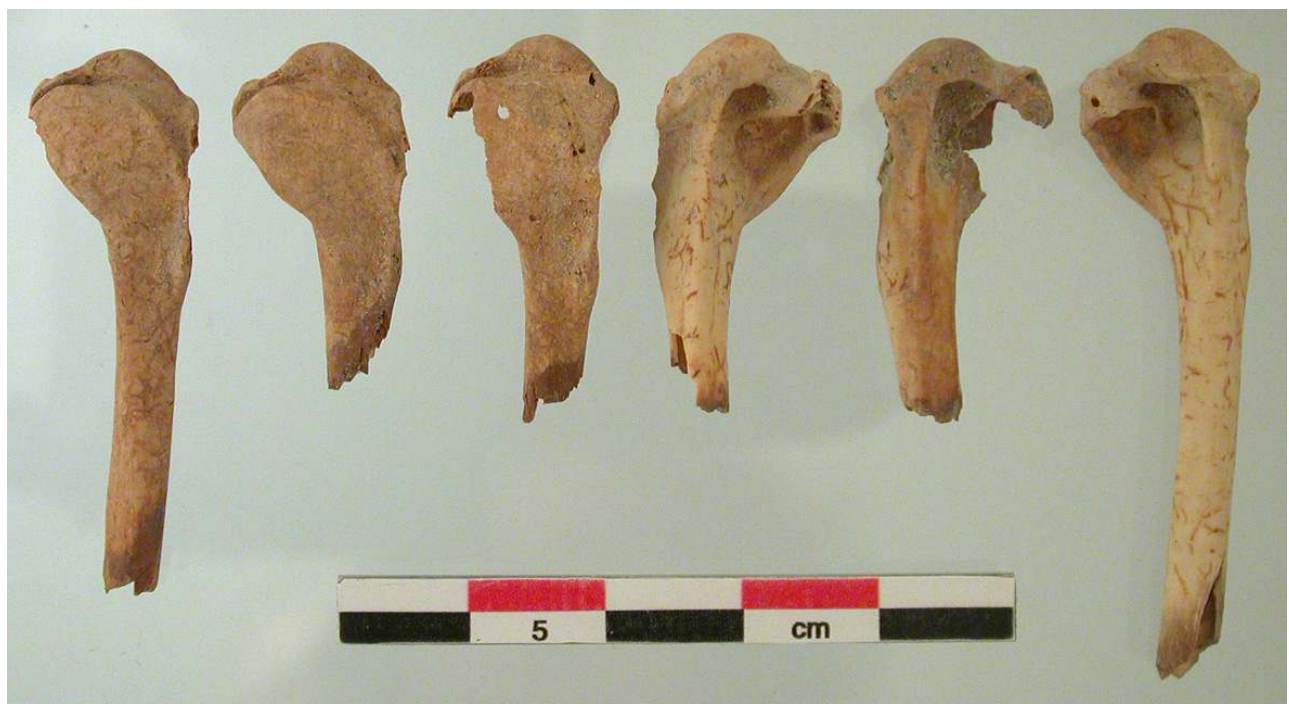

Humérus (ailes) de sterne. Les extrémités distales sont cassées sur os frais et présentent des traces de brûlures directes indiquant que les ailes ont été brisées avant de faire rôtir les oiseaux directement sur le feu.

(c) V. Laroulandie.

Mais si les fouilles archéologiques ont permis d'apporter quelques réponses, elles posent aussi de nouvelles questions.

La gestion des morts reste la grande inconnue. Au moment du naufrage, même si les requins ont pu être des auxiliaires efficaces, les Français se sont trouvés confrontés à un grand nombre de cadavres. Ne serait-ce que la trentaine d'esclaves décédés les premiers jours. Par rapport aux pratiques de l'époque et aux notions hygiénistes déjà en vogue, il est probable qu'on leur a donné une sépulture. Mais les textes n'en parlent pas et nous ne les avons pas retrouvées. Après le départ des Français, les naufragés ont pu gérer leurs morts eux-mêmes. Bien sûr les morts constituent un apport calorique non négligeable, surtout en condition d'isolement extrême. Cependant, sur les $200 \mathrm{~m}^{2}$ de niveaux archéologiques très riches en restes consommés, aucun fragment d'os humain n'a été trouvé. Les restes de deux individus ont été trouvés dans les niveaux de déblais liés à la construction de la station météorologique. Ils ne montrent aucune trace ni de violence ni de décharnement. S'agit-il des dépouilles mortelles des naufragés de L'Utile? Sans aucun doute. D'autres éléments provenant du navire (une boucle de chaussure, une garde d'épée...) ou transformés par les naufragés (une série de récipients en cuivre, deux bracelets en cuivre) ont aussi été retrouvés dans ce même niveau. Après le cyclone de 1956 et la destruction des premières installations météorologiques, Météo France prend la décision de construire deux bâtiments semi-enterrés. Ces bâtiments sont localisés au milieu de l'habitat des naufragés et s'appuient autant que possible sur les vestiges de murs du XVIII ${ }^{e}$ siècle. À cette occasion, les niveaux archéologiques ont été recoupés par les ouvriers et les vestiges rencontrés déplacés. Comment ont-ils pu déplacer puis rejeter deux individus sans que cela ne s'ébruite? Et, interrogation plus fondamentale: où et comment étaient déposés ces individus? Autant d'informations perdues à jamais.

Une autre interrogation concerne la découverte d'un bâtiment construit et occupé entre 1776 et 1953 (fig. $\mathbf{n}^{\circ} \mathbf{8}$ ). Le mode de construction est quasiment identique à celui des bâtiments édifiés par les naufragés de l'Utile, excepté une largeur de mur moindre. Le 
remplissage de cette occupation est plus foncé (presque noir) et renferme, en plus de restes de sternes, une assez grande quantité de poissons d'assez grande taille. Le mobilier ne comporte aucun élément qui ne pourrait être attribué aux naufragés de l'Utile. Et pourtant, stratigraphiquement, cette occupation se situe, avec certitude, après le sauvetage des Malgaches et avant l'arrivée des météorologues.

Figure 8

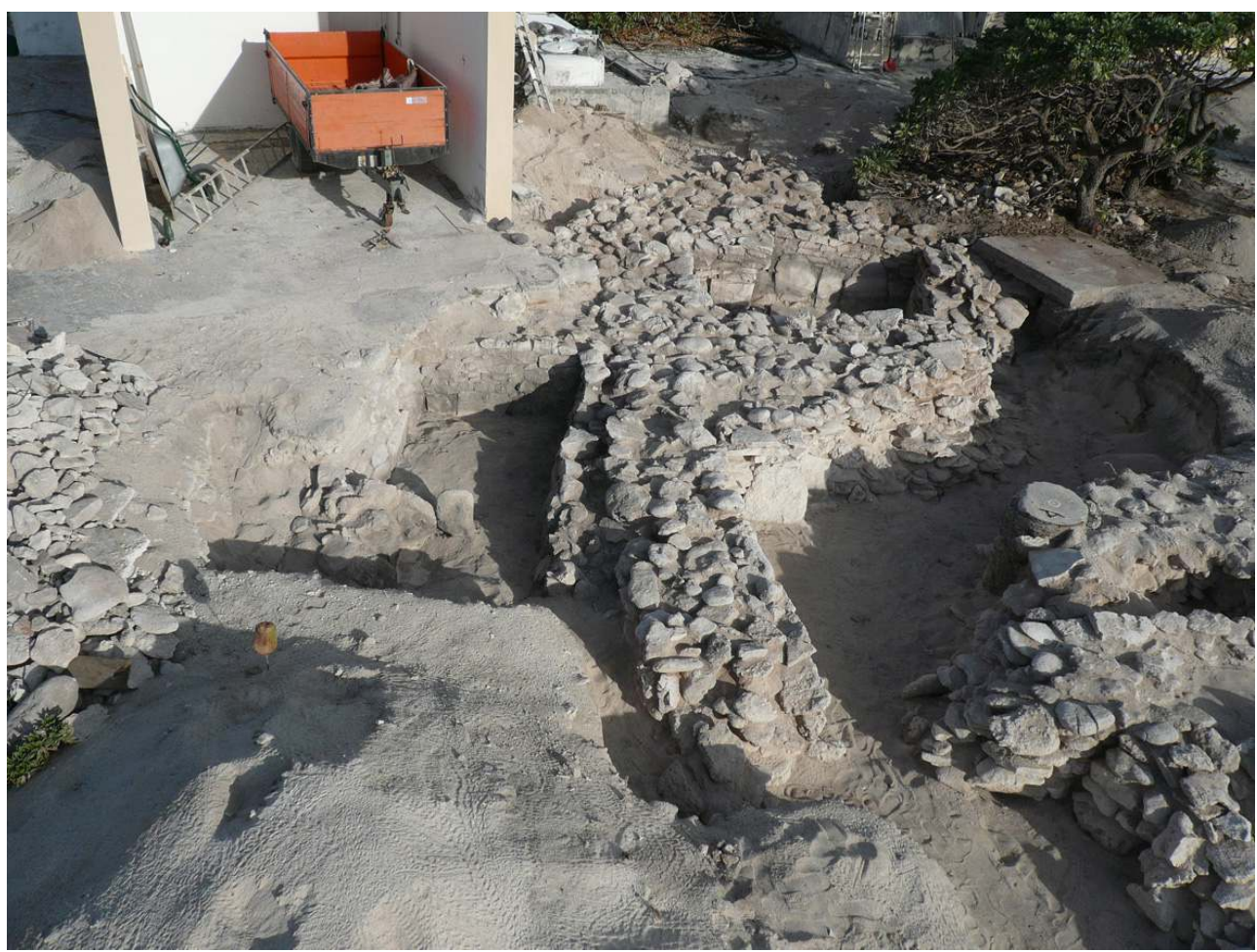

Les bâtiments mis au jour en 2010. Sur la gauche, le bâtiment 15, construit après le départ des naufragés et avant la construction de la station météo.

(c) J.-F. Rebeyrotte-Gran.

19 Tromelin est un lieu de mémoire difficilement accessible. Les vestiges matériels laissés par les naufragés de l'Utile constituent une image statique d'un événement dynamique. Il appartient aux archéologues d'essayer d'en décoder et d'en déchiffrer la signification, afin de restituer la réalité quotidienne des naufragés et d'alimenter ainsi la mémoire historique de l'événement. Alors que celle-ci s'estompe avec le temps, les vestiges matériels sont comme une bouée de sauvetage à laquelle peut se raccrocher la mémoire. Le patrimoine qui concerne l'esclavage est particulièrement rare et fragile. Il nous faut donc non seulement prendre garde de ne pas le détruire, mais pour le moins, en faire une lecture et un enregistrement attentifs. Le devoir des archéologues est de restituer les données recueillies. Il s'agit là d'une étape indispensable au partage des connaissances, faute de quoi tous les efforts consentis auraient été vains. 


\section{NOTES}

1. - Manuscrit anonyme (SHD Marine, Lorient - 1P297, liasse 14, pièce 85) attribué par Max Guérout (2010) à l'écrivain du bord de l'Utile, Hilarion Dubuisson de Keraudic qui constitue un journal non officiel, succinct mais précis, des événements.

2. - Intitulé « Relation des principales circonstances qui ont accompagné \& suivi le naufrage de la Frégate l'Utile, Capitaine M. de Lafargue, sur le réssif de l'isle de Sable ou du Corail, situé au $15^{\mathrm{e}}$

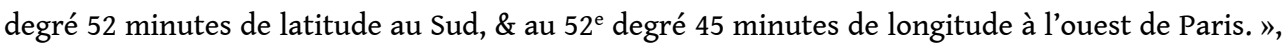
non daté, imprimé à Bordeaux chez Jean Chappuis, trouvé par Bernard Harnie-Cousseau à Bayonne dans les archives de la famille Van Huffel.

3. - Lettre de M. Maillart, Intendant des Isles de France et de Bourbon à Monsieur de Sartines, Ministre de la Marine, datée du 16 décembre 1776 (A. N. Marine G222 - f³4 ${ }^{\mathrm{B}}$ ) : «[...] et qu'ils avaient laissé sur l'Ile un grand nombre de noir javas. ».

4. - La synthèse des deux premières missions est présentée dans l'ouvrage : GUEROUT, Max et ROMON, Thomas. Tromelin, l'île aux esclaves oubliés. Paris : CNRS Édition/Inrap, 2010.

5. - Voir par exemple: ACQUIER J.-L. et HARIJAONA R. Architectures de Madagascar. Paris: Arthaud-Berger-Levrault, 1997.

\section{RÉSUMÉS}

Le naufrage de l'Utile, le 31 juillet 1761 sur l'île de Tromelin et le sort réservé à sa « cargaison » : des esclaves transportés en fraude, nous invitent à nous interroger sur la notion de mémoire d'un tel événement, ainsi qu'à son évolution dans le temps. Cette mémoire est intimement liée à la présence de vestiges matériels qui ont un rôle de support. L'enfouissement, au fil du temps, des traces laissées par les naufragés gomme leur réalité quotidienne et en provoque une quasiamnésie. S'inscrivant dans les projets La route de l'esclave et Les anneaux de la mémoire, les fouilles archéologiques menées par le Groupe de Recherche en Archéologie Navale, avec la collaboration de l'Institut National de Recherches Archéologiques Préventives, ont permis de mettre au jour les restes de leur quotidien. Elles participent ainsi à la construction de la réalité historique de cet événement.

The shipwreck of l'Utile, on the 31st July 1761 on the island of Tromelin, and the fate reserved for its 'cargo' of illegally transported slaves, led us to probe the memory of the event, and its evolution in time. This memory is closely related to the presence of material remains, which provide insights into the events. The gradual burial, with time, of the traces left by the castaways erases the evidence of their daily reality. Within the framework of 'La route de l'esclave and Les anneaux de la mémoire' archaeological excavations carried out by the Groupe de Recherche en Archéologie Navale, with the collaboration of the Institut National de Recherches Archéologiques Préventives, allowed to uncover the remains of the survivor's daily lives. These shed new light on the historical reality of this event. 
INDEX

Mots-clés : traite, esclavage, naufrage, Océan Indien, archéologie

\section{AUTEURS}

\section{THOMAS ROMON}

Institut National de Recherches Archéologiques Préventives (Inrap, route de Dolé, 97113 Gourbeyre) et UMR 5199 (Pacea, Laboratoire d'Anthropologie des Populations du Passé et du Présent, Université de Bordeaux I), chargé des fouilles archéologiques terrestres thomas.romon@inrap.fr

\section{MAX GUEROUT}

Groupe de Recherche en Archéologie Navale (GRAN, Service historique de la Défense, rue de l'ancienne Corderie, BP 45, 83800 Toulon Naval) et Laboratoire d'Histoire et d'Archéologie Maritime FED 4124 (Paris IV Sorbonne - Musée de la Marine), chef de la mission « l'Utile... 1761, esclaves oubliés »mrgueroutmaxadrien@9business.fr 